POINTS OF VIEW

\section{Axes, ticks and grids}

\author{
Make navigational elements distinct and \\ unobtrusive to maintain visual priority of data.
}

Figures that present large amounts of quantitative information can be more accurately assessed when complemented with effective axes, ticks and grids. These navigational elements provide scale and aid in accurate assessment of lengths and proportions.

Navigational cues must be distinct from the figure's primary information. The Gestalt principles ${ }^{1}$ inform us how to use line width, color and transparency to achieve this (Fig. 1). At all times, keep the datato-ink ratio high by using the least amount of ink for navigational elements.
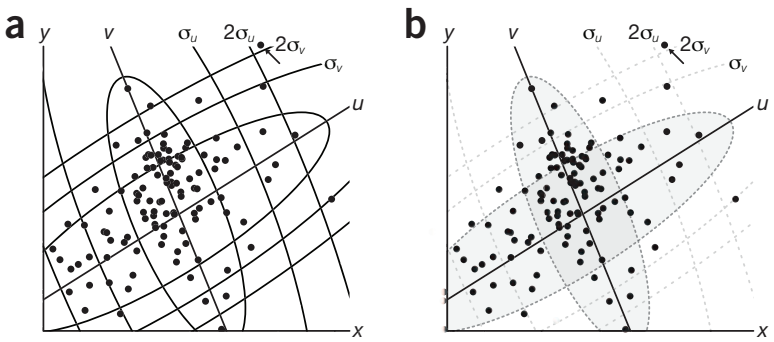

Figure 1 | Retain the salience ${ }^{3}$ of primary data by making navigational elements visually distinct. (a) Gestalt principles of ground and similarity describe why we have difficulty visually organizing information in a figure in which the same line style is used for different purposes. (b) Visual layers are established by assigning different thickness and style to axes, contours and cluster boundaries.

If your data have a coordinate system, the figure's axes are the foundation and are critical in orienting the reader. Axis weight should be modest $-0.5 \mathrm{pt}$ is sufficient - and unless the figure is particularly large, you should avoid bounding it by axes on all sides. This containment is often mistaken for organization, which can be otherwise achieved by a suitable amount of negative space. Refrain from placing arrows on axes-their orientation is almost never in doubt. Multipanel figures should maintain fixed scales when possible to facilitate comparison because variation in nondata components,

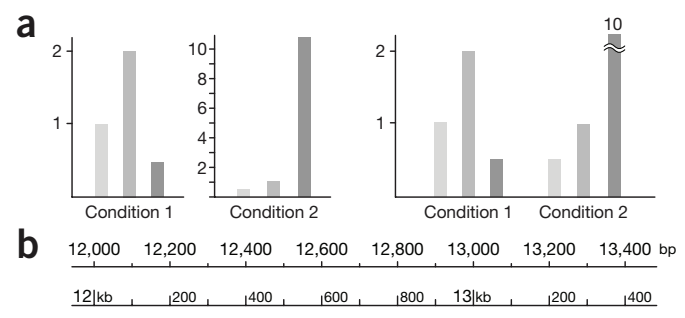

Figure 2 | Avoid unnecessary variation and repetition in axes and ticks. (a) If absolute differences are important, maintain axis scaling across panels. Draw a single $y$ axis to emphasize that the scale is fixed. In bar plots, use breaks to shorten outlier elements that would otherwise compress the dynamic range of the data. (b) Duplication of nonsignificant digits in tick marks should be reduced or removed altogether by adjusting the units. a

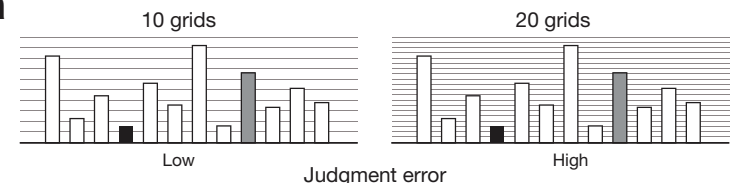

b
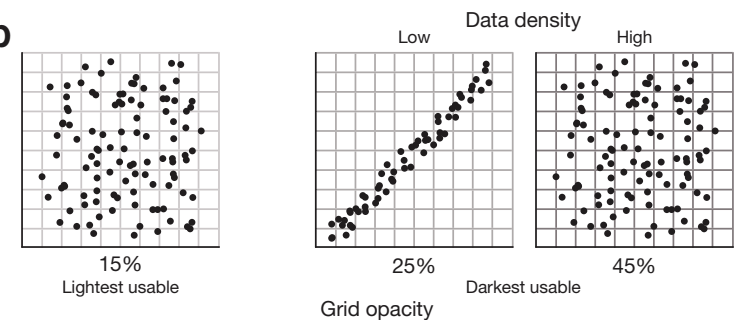

Figure 3 | Control grid density and transparency to maintain separability from data and other grids. (a) User studies in which readers were asked to assess the proportion of bar heights show that the density of grid lines is correlated with judgment error ${ }^{4}$. (b) Grid line transparency is most effective in the range of $15 \%-45 \%$, depending on data density 4 .

such as axis ranges, can be easily overlooked (Fig. 2a). Make sure that outliers do not compress the dynamic range of the bulk of your datause bar or axis breaks, but always be sensitive to the journal's policies on breaks and the fact that these elements can disguise important patterns.

When they are densely labeled, axis ticks burden the figure with repetition. This applies specifically to views of data across large genomes, which are filled with repeating nonsignificant zeros. Innovative strategies exist ${ }^{2}$ to keep tick label complexity low while maintaining usability (Fig. 2b).

Grids are used to establish sight lines to compare proportions and relate positions to axis ticks. The number of grids powerfully suggests the scale at which differences are important. Faced with a dense grid, the readers will conclude that they should pay close attention to minor fluctuations in the data and infer that the degree of uncertainty is low. Do not send this message falsely. Furthermore, dense grids impede accurate judgment, as tracing them to their axis labels is confounded by increased density (Fig. 3a). Ultimately, no grid may be better than a badly chosen one-use a grid when needed rather than by default.

Patterns in data can be quickly obscured if too much ink is used for grids. The grid should be dark enough to be seen clearly (anticipate that LCD projectors will wash out light colors) but not so dark as to appear as a fence in front of data. A useful guideline is to use $15 \%$ as the minimum grid opacity. Maximum opacity should be $25 \%-45 \%$, in proportion to the figure's data density (Fig. 3b).

A common example of functional layering of information modalities is found in maps. The next time you navigate a map, take note of the strategies used by the cartographer to generate a hierarchy of meaning between place names, terrain type, elevation and landmark annotations, as you relate these features to elements in your figures.

\section{Martin Krzywinski}

\section{COMPETING FINANCIAL INTERESTS}

The author declares no competing financial interests.

1. Wong, B. Nat. Methods 7, 863 (2010).

2. Pak T.R. \& Roth, F.P. Bioinformatics 29, 384-386 (2013).

3. Wong, B. Nat. Methods 7, 773 (2010).

4. Heer, J. \& Bostock, M. in Proc. CHI Conf. Hum. Factors Comput. Syst. 203-212 $(A C M, 2010)$.

Martin Krzywinski is a staff scientist at Canada's Michael Smith Genome Sciences Centre. 